\title{
SUGGEST AN INTELLIGENT FRAMEWORK FOR BUILDING BUSINESS PROCESS MANAGEMENT
}

\author{
Almutairi Raed, Dr. Djamal Ziani \\ Department of Information Systems College of Computers and Information Sciences \\ King Saud University, Riyadh, KSA
}

\begin{abstract}
As companies enter into the digital world, information technology is playing a major role in bringing process improvements to the forefront of business management. In the recent decades, many organizations have struggled to redesign and improve their business processes to reduce their total cost. The main contribution of this research study is to propose an intelligent framework that possesses the ability to employ a database of best practices, business standards, and business activity history in order to permit the manager to analyze and improve the design of the business processes.

In addition, the other objective of this research is to build a business process or workflow directly from its process design logic in order to enable rapid process development and deployment. This procedure requires some technical improvements of the business design, as it is mainly based on building the business process using Microsoft Office Visio, which communicates the defined business process to the business process management engine.
\end{abstract}

\section{KEYWORDS}

Business process improvements, business process intelligence, business process implementation

\section{INTRODUCTION}

Business process can be defined as the composition of different activities working together to achieve an organizational goal [1]. Implementing business process redesign should consider several best practices, such as time, quality, cost, and flexibility, in terms of how and when to implement business process management (BPM) [2].

The main contribution of this research study is to propose an intelligent framework that possesses the ability to employ a database of best practices, business standards, and business activity history in order to permit a manager to analyze and improve the design of the business processes.

In addition, the other objective of this research is to build a business process or workflow directly from its process design logic in order to enable rapid process development and deployment. This procedure requires some technical improvements of the business design, as it is mainly based on building the business process using Microsoft Office Visio, which communicates the defined business process to the business process management engine.

Another purpose of this research work is to estimate all costs for each business process before and after applying the optimization in order to assess the overall cost. In addition, a standard duration DOI : 10.5121/ijsea.2015.6106 
International Journal of Software Engineering \& Applications (IJSEA), Vol.6, No.1, January 2015

will be allocated to each phase in order to alert the business user when he has spent the time that had been allocated to complete that particular task. The results of our research permit an organization to accelerate processes and increase their efficiency by reducing costs of processes, as well as to increase service quality and dependability.

\section{RELATED WORK}

Business process activity needs to be analyzed, whether in sequence or in parallel, to remove any delays in the process itself. One major thing that researchers have considered is the organization policy [1], which should to be taken into account before applying process improvements. Also, some questions have been raised by researchers regarding the gathering of business process data to suggest some improvement guides.

The authors in [2] suggested the Use of the business process data mining technique in order to improve the business processes. This technique has been applied in a Brazilian software company, which demonstrated that this technique was extremely useful. However, the main drawback of using this technique is that this framework is dependent on the log file, which does not provide an instant result, and this log file also needs to be cleaned and extracted regularly.

Managers, directors, process owners, and analysts should work in collaboration as one team to brainstorm ways to redesign an alternative workflow [3]. Then, they should apply the new workflow to a real-life setting to evaluate it. This has a major impact on the workflow redesign procedure, but still it depends on the human factors and does not involve real data extracted from activity within the workflow.

The authors in [4] provided a set of measurements with associated scores; these scores are to be written by the team members, who can be either internal or external performers. This technique is basically dependent on conducting several meetings to draw a roadmap for the organization. However, these procedures will take more time and do not mainly utilize information technology extracted from work activity.

The proposed framework in [5] includes four layers: information acquisition, performance evaluation, structural defects identification, and improved model generation. Each layer has its own technique, but this paper did not provide the methodology in detail for each layer.

There are some papers that suggested that each type of stakeholder should have their own judgment from their own perspective [6], but those papers described the evaluation and data analysis at a high level without going deeper into the technique used in the paper. Also, they suggested that stakeholders should do some monitoring processes and pay attention to those that exceed their time limits, without going into further details on how to do so. In addition, they used business process management notation (BPMN) as the standard representation, and they suggested prioritizing the activity to achieve the optimal cost of the process.

The authors in [7] suggested business process intelligent (BPI) should be utilized to support business process improvement instead of spending more time cleaning and analyzing log files. In addition, they suggested a mechanism to transform historical data using extraction, transformation, and loading (ETL) to solve or optimize the resource assignment problem. Thus, the main features of BPI are to optimize workflow design and resource assignment problems. 
International Journal of Software Engineering \& Applications (IJSEA), Vol.6, No.1, January 2015

This paper [8] illustrated that the organization components such as strategy, structure, processes, technology, and people (roles and skills) should be well integrated to accomplish an overall business improvement. In addition, the organization components should depend on human factors to make the design of a business more logical instead of depending on artificial intelligence, which will be more costly.

This paper [9] explained the importance of designing a business process that depends on standardization methodology. Standardization should be involved in the design from the lowest layer, the database level, to a higher level of designing business processes. Standardization can be viewed from two perspectives: manufacturers' perspective and customers' perspective. From the manufacturer's perspective, this will decrease the overall cost of developing and designing systems; from a customer's view, this will meet his good expectations of the product.

The process of achieving enterprise integration includes all managerial and technical factors that enable cross functional process integration [10]. The result is a customer-oriented management structure, with information systems, formally related to the processes and integration of procedures required to establish and retain customer satisfaction. It is very necessary to define an expert leader and to conduct a meeting in order to define "as-is" and "to-be" phases.

Business intelligence has become extremely essential to organizations [11]. Due to the globalization of the world market and massive advances in information technology through which businesses gather, organizations are able to transform and then analyze data to predict the future of the business to enhance and provide better decision results.

\section{Proposed Framework}

\subsection{Proposed architecture}

The purpose of this solution is to find the most suitable way to maximize employee performance. To achieve this, our proposed intelligent framework will analyze and monitor activities and "tasks" inside the business process workflows. A trigger will be added to notify or alert the user about an activity's deadline. To accomplish this operation, we need to identify a time limit for each phase or activity within a process. To do so, we will design and implement an intelligent framework that will act as an enabler for process owners to monitor and trigger the durations of different processes and activities, as well as to seek any improvement opportunities. The proposed framework system can be utilized as a new generation of business process management improvement by identifying and measuring phase limits while users continue to use the system.

In accordance with the specified requirements, we have put together a plan to describe how the system components should interact. Figure 1 shows the main system components and how they are linked to one another. At the beginning, we need to create the workflow design using Microsoft Visio with the BPMN standard; then, the system engine will parse the design file and will validate and verify the workflow design. Afterwards, the engine will automatically create the workflow process and its related objects. There are two main components of system architecture, which are described below.

- XML Design file

- Business process engine 


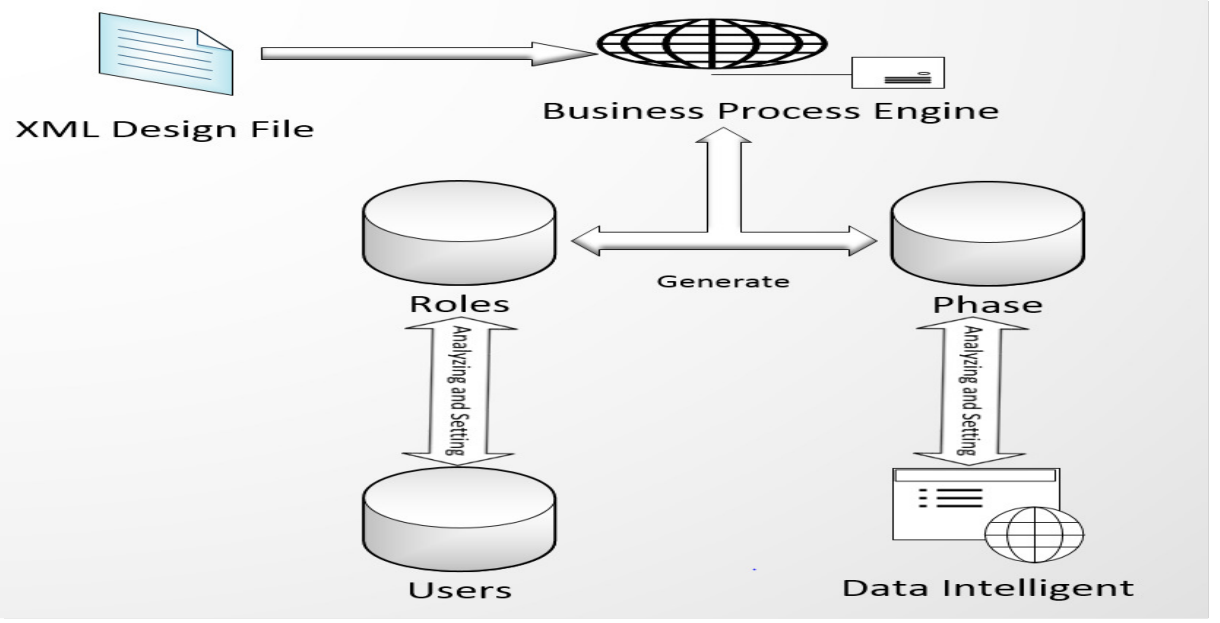

Figure 1: System architecture

\subsubsection{XML Design file:}

The suggested system has used Microsoft Visio to draw objects-such as the entity, task, and arrow-that are based on the BPMN standard. Microsoft Visio is "a diagramming tool that can be used to visually communicate technical as well as non-technical representations of ideas, processes, concepts, structures, layouts, software models, blueprints, etc." [2]. Microsoft Visio stores files in the VSDX format that is based on XML on the backend. These files contain the workflow design, which stores object data such as entities and connectors, in Extensible Markup Language (XML). This will allow developers to manipulate and work with these files programmatically because it is based on XML structured technology, as shown in Figure 2.

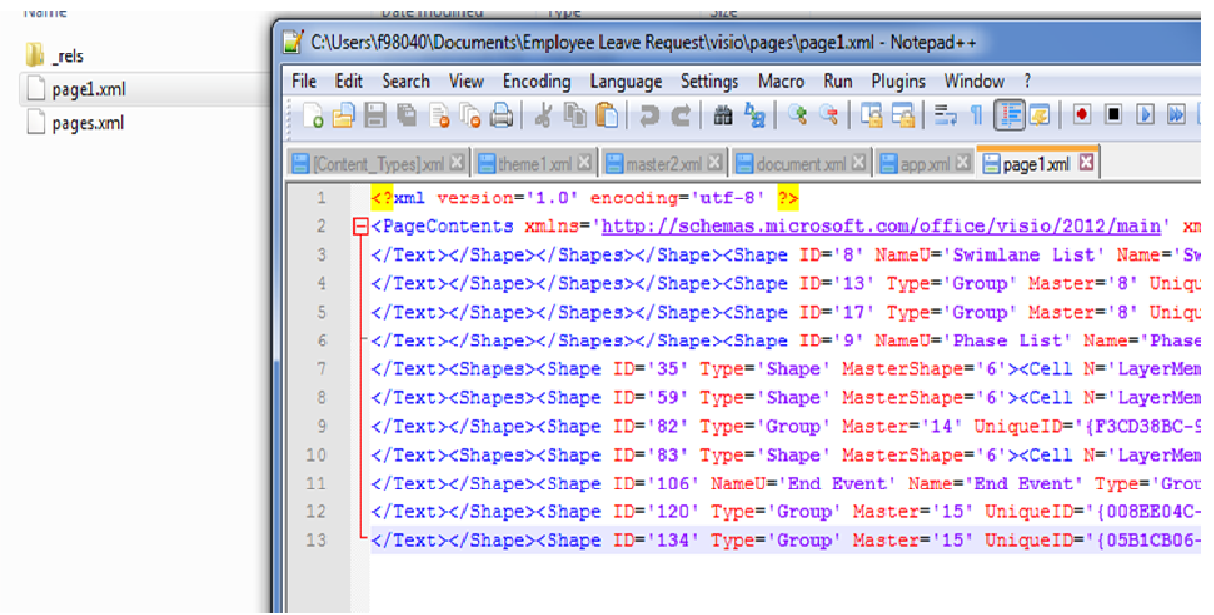

Figure 2: Extracted VSDX File 


\subsubsection{Business process engine:}

This part of the proposed system is responsible for converting XML file design into three main objects: connectors, roles, and phases ("tasks" or "activities"). Each object from these extracted objects will be explained in the next section. After the developer writes the main process properties, such as the name and the process code or reference, the engine will generate these objects from the uploaded design file. Once this process has been completed, a log will show the results of generating these objects, as shown in Figure 3. In addition, the engine is responsible for moving process instances and workflow from one phase to another, and for executing any phase based on its respective type.

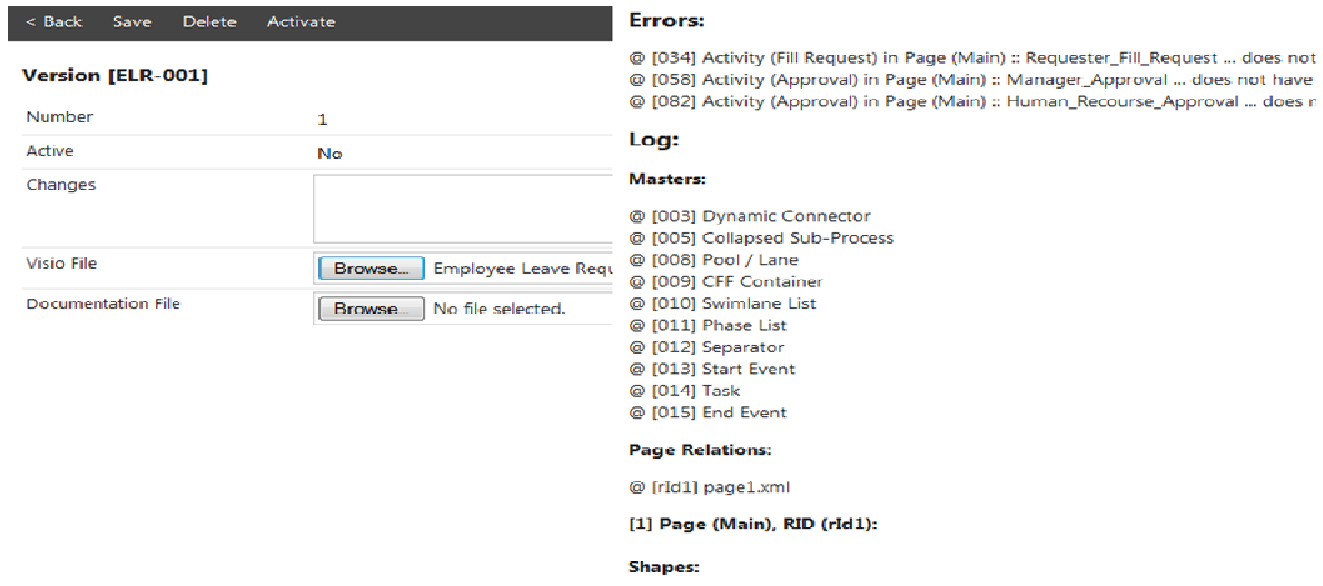

Figure 3: Log file generated from Process Engine

From the log file, the engine will interpret the main objects that are a concern to the proposed system; for example, it will extract the following object types from the design file:

- Errors

- Masters

- Page Relation

- Connectors.

\section{a) Errors:}

If the log contains any potential errors, it will be shown in this section of the generated $\log$ file.

b) Masters:

This will list all main objects except the connectors, such as activities, groups, and events (if there are any events within the design).

c) Page relation:

This part will list all related pages extracted from the file.

\section{d) Connectors:}

These are used to extract all connections between different activities; the engine uses the connectors as a path between workflow activities. 
International Journal of Software Engineering \& Applications (IJSEA), Vol.6, No.1, January 2015

\subsection{System Unique Functionalities}

\subsubsection{Rapid process development}

A business process consists of different activities done in unison to achieve a common goal. As mentioned before, one objective of this research was to find a way for the process designer to build a business process or workflow directly from the process design logic in order to enable rapid process development and deployment.

The main objective of rapid process development is to make the business process engine able to translate the process design into business process objects. This design is based on the BPMN2 standard model using a tool like Microsoft Visio. It will parse the design as an input and generate the business process objects, such as the process phases, roles, and connectors, as an output.

\subsubsection{Best practices}

Best practices methodology has been gathered and implemented in many different areas including health care, manufacturing, and software development. From a business process perspective, best practices can be described as the redesign of a business process that is derived from many organizations in order to overcome a technical business process design challenge [13]. As in the proposed framework, each workflow can links to another workflow as a result of the best practice for that process; in addition, a category object has been created for each process to specify which type category that process belongs:

a) Department Process Category: This process does not follow the best practice procedure. This might because it is a very specific procedure specifically for a department.

b) Best Practice Process Category: This category follows the best practice standards among the best similar organizations.

Once best practices for a department process have been created, a pointer will be created for a best practice to indicate this best practice process to be the outcome of non-best practice process that will be improved over time.

\subsubsection{Process Standard}

Process standard will allow the organization business process to increase readiness to the employee and outsourcing company in order to improve overall employee performance. A process standard has been implemented as an object in the proposed framework; this standard object can be assigned for each phase or activity within a process to define the timeline for each task. Mainly, as shown in Figure 4, we embedded the standard in the design of the framework; in addition, there are three main attributes for each phase that we have used to set the process standardization. 
International Journal of Software Engineering \& Applications (IJSEA), Vol.6, No.1, January 2015

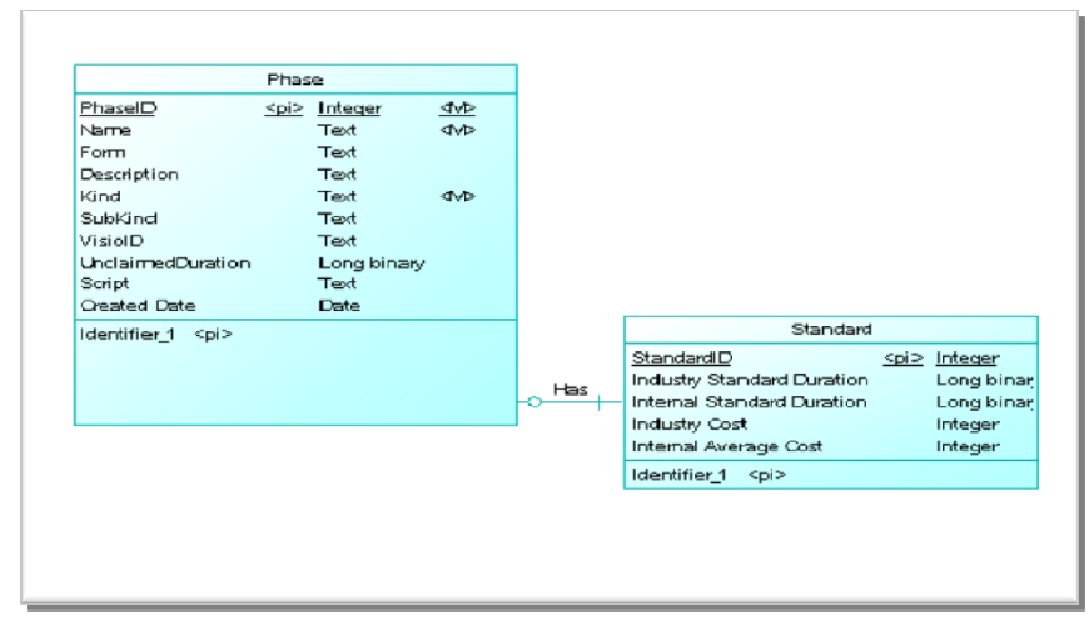

Figure 4: Embedded standard in the design of the proposed framework

\section{a) Internal "local" standard}

In this scenario we have applied a phase standard to be derived from an internal organizational unit, or from a department that should follow any internal policies or an organizational policy. Such policies have an official form that specifies the maximum duration allowed for each phase to be completed. Thus, the maximum duration will be set when the process has been implemented.

\section{b) Industry standard}

In this scenario, we need to measure what the standard durations and phases are that are derived from leading organizations and that any organization should follow to obtain an official certificate, such as ISO 10001. Many papers have shown that applying a standard duration will reflect on the organization's performance by reducing its cost [14].

\section{c) Average standard}

This scenario is based completely on the system engine calculating the average duration and using this value to set the maximum duration of each phase. If the user passes this time allowance, the system will alert him using some indicator such as changing the color of that phase or sending him an email to remind him about it.

\section{Evaluation}

The quantitative research approach has been used to validate the proposed framework. A sample of 21 users representing different roles of users was evaluated. The study was conducted at one of the leading health care provider organizations of Saudi Arabia. A sample of three categories was defined, representing two main groups commonly associated with business process stakeholders and developers. These groups consist of participants who have had a primary role in business processes. On the other hand, the participating developers were usually business analysts having direct contact with main stakeholders of business processes. These participating groups were provided the necessary overview of the proposed system's unique functionality, including process 
International Journal of Software Engineering \& Applications (IJSEA), Vol.6, No.1, January 2015

best practice, process standard, and rapid process development, as well as basic instructions on the use of the proposed framework.

Having gathered committed participants who interacted with business process systems and best represented the targeted sample groups, the final sample pool eventually comprised of the following percentage breakdowns. The users of business processes consisted of 17 participants. The developers' group consisted of 4 participants.

Those users were provided the necessary access to the basic instructions and they were viewed the functionalities of the proposed framework. After sometime, the group was requested to respond to an online questionnaire based upon their relationship to business process systems and business users. The survey consisted of 4 questions, each on a 5-responses scale which is:

$$
\begin{array}{ll}
\text { - } & \text { Strongly agree. } \\
\text { - } & \text { Agree. } \\
\text { - } & \text { Neutral. } \\
\text { - } & \text { Sisagree. } \\
& \text { Strongly disagree. }
\end{array}
$$

\section{A. System efficiency and overall cost savings}

The first question we asked of participants was related to "system efficiency and overall cost savings". This primarily referred to those savings experienced by the employee, manager, and overall organization. More specifically, it related to the savings in terms of both personal time and financial costs. $56 \%$ of participants surveyed agreed with this statement and $19 \%$ was neutral while $14 \%$ of participants were strongly agreed "Figure 5 ".

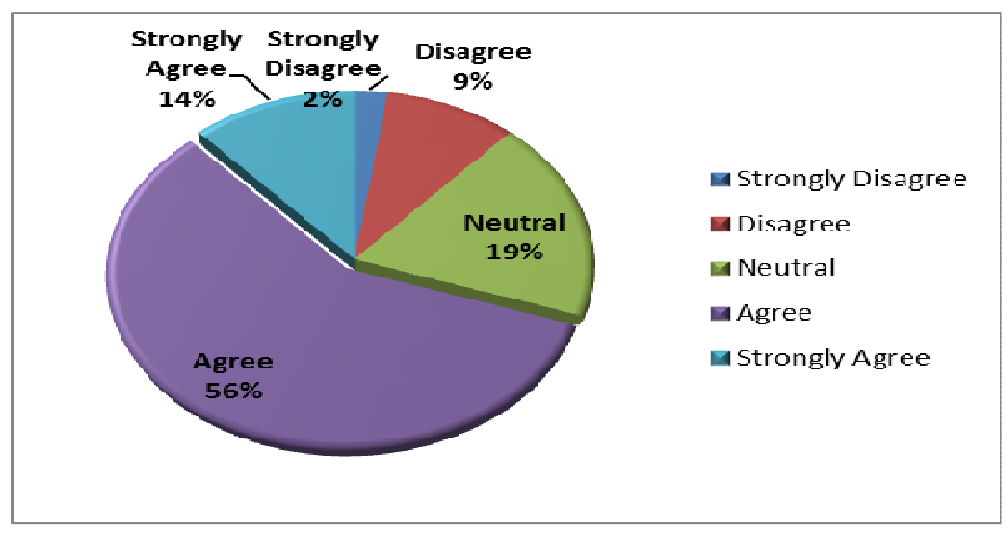

Figure 5: System efficiency and overall cost savings

\section{B. Optimization of building and implementing business process management components such as work activities, phases, and data flow by accelerating the development time for business processes}


International Journal of Software Engineering \& Applications (IJSEA), Vol.6, No.1, January 2015

We asked participants to what extent they agreed with the following statements "The Suggested framework help to optimize of building and implementing business process management components such as work activities, phases, and data flow". Agreed 54\% of participants surveyed agreed with this statement and $20 \%$ was strongly agreed while $9 \%$ of participants were neutral "Figure 6".

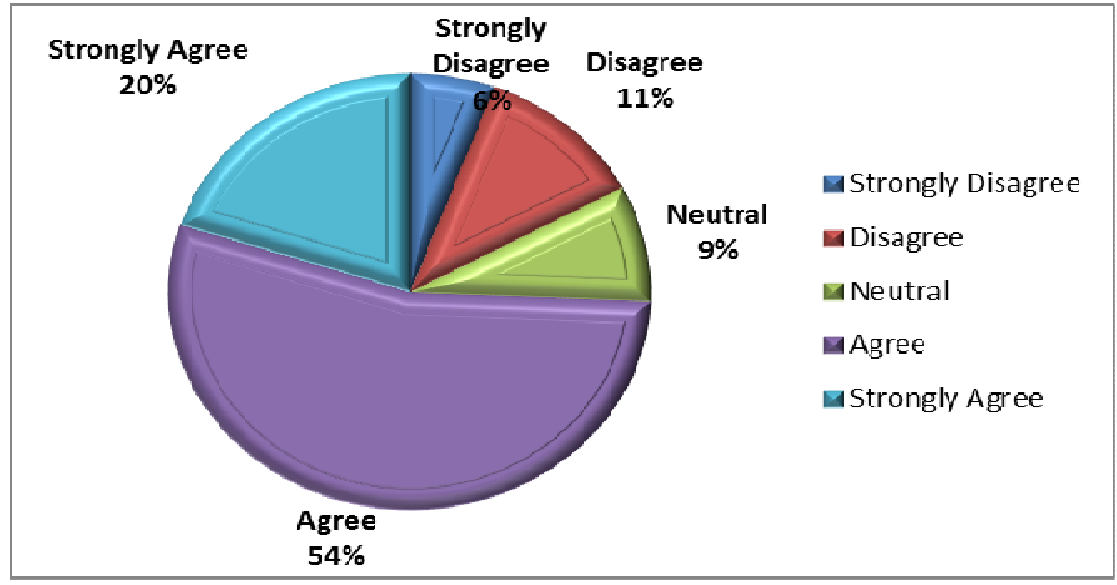

Figure 6: Optimization of building and implementing business process management

\section{Influence on the design of business processes by considering the best practice.}

The third factor explored in the questionnaire was the effect of considering how the best practices for business process can positively influence the design of business processes. This main focus here was to focus on any improvements in time, quality, or employee satisfaction that can be made to any business process design by applying the best practices that have previously described. $57 \%$ of participants surveyed agreed with this statement and $21 \%$ of participants were neutral and $12 \%$ of participants were strongly agreed "Figure 7".

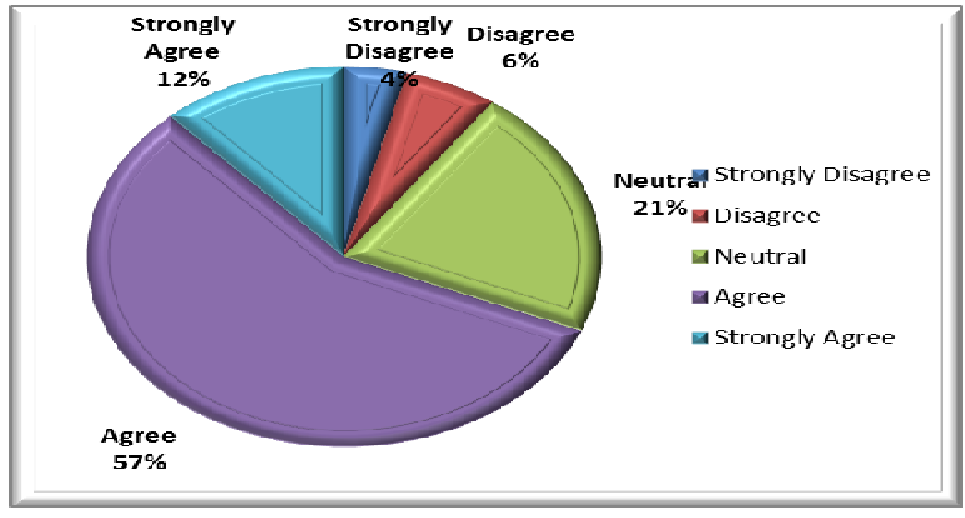

Figure 7: Influence on the design of business processes by considering the best practice 


\section{Usability}

To validate the performance of the system usability, the following three terms were used to evaluated together the subjective aspect of end-user experience: (1) user-friendliness, (2) reliability, and (3) value added. 33\% of participants surveyed were neutral with this statement, while $33 \%$ agreed and $22 \%$ of participants were strongly "Figure 8 ".

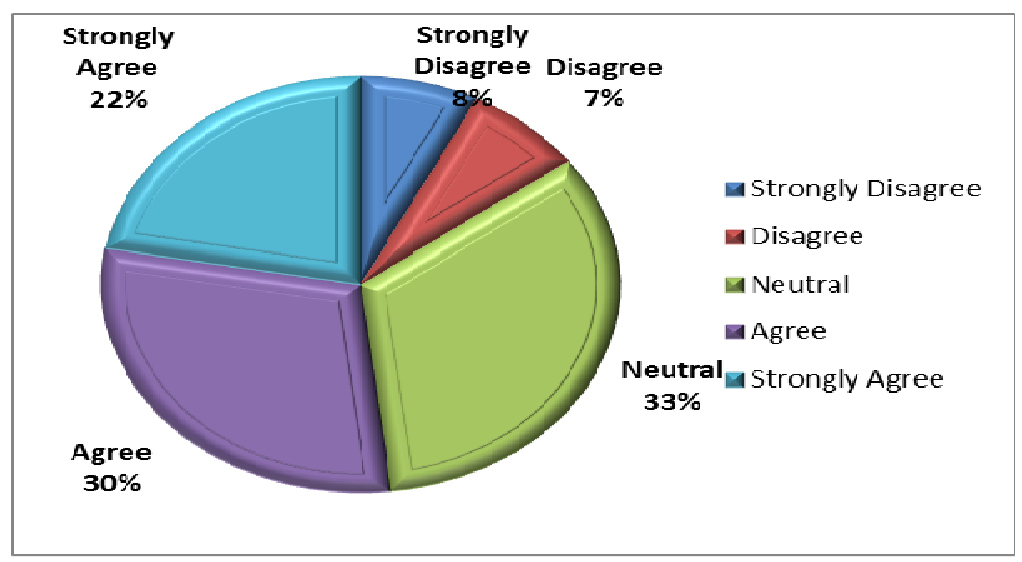

Figure 8: System usability

\section{CONCLUSION}

The main goal of this research work was to provide an intelligent business process that had a positive impact on an organization processes, and to redefine the traditional approach to business processes in an organization. We proposed a business process framework to improve communications in order to reduce the cost, increase the effectiveness and the efficiency, and increase the usability among the developers and business users.

\section{REFERENCES}

[1] MD. Maaz, Manish Kumar, (2012) "Structured Method for Business Process Improvement". Third International Conference on Services in Emerging Markets 2012.

[2 ]Lemos, A.M.; Sabino, C.C.; Lima, R.M.F.; Oliveira, C.A.L., (2011) "Using process mining in software development process management: A case study," Systems, Man, and Cybernetics (SMC), 2011 IEEE International Conference on , vol., no., pp. 1181,1186, 9-12 Oct.

[3] Amiyo, M.; Nabukenya, J.; Sol, H.G.,(2012 "A Repeatable Collaboration Process for Exploring Business Process Improvement Alternatives," System Science (HICSS), 2012 45th Hawaii International Conference on , vol., no., pp.326,335, 4-7 .

[4] Asato, R.; de Mesquita Spinola, M.; Costa, I.; de Farias Silva, W.H., (2009) "Alignment between the business strategy and the software processes improvement: A roadmap for the implementation," Management of Engineering \& Technology, 2009. PICMET 2009. Portland International Conference on , vol., no., pp.1066,1071, 2-6 Aug.

[5] Liang Chen, Tao Xue, Ali Yang, (2009)"Business Process Continuous Improvement System Based on Workflow Mining Technology," csie, vol. 5, pp.414-418, 2009 WRI World Congress on Computer Science and Information Engineering. 
International Journal of Software Engineering \& Applications (IJSEA), Vol.6, No.1, January 2015

[6] Lodhi, A.; Koppen, V., (2011) "Business process modeling for post execution analysis and improvement," Software, Knowledge Information, Industrial Management and Applications (SKIMA), 2011 5th International Conference on, vol., no., pp.1,8, 8-11 Sept. 2011

[7] Sohail, A.; Dominic, P.D.D., (2012)"A gap between Business Process Intelligence and redesign process," Computer \& Information Science (ICCIS), 2012 International Conference on , vol.1, no., pp.136,142, 12-14 June 2012 .

[8] Paul L. BannermaNICTA, Sydney, (2009) "Capturing business benefits from process improvement: four fallacies and what to do about them", Proceedings of the 6th International Conference on Frontiers of Information Technology Abbottabad, Pakistan. December 16-18, 2009.

[9] Harshavardhan Karandikar, Srinivas Nidamarthi, (2007) "Implementing a platform strategy for a systems business via standardization", Journal of Manufacturing Technology Management, Vol. 18 Iss: 3, pp. $267-280,2007$.

[10] Douglas W. Frye, Thomas R. Gulledge, (2007) "End-to-end business process scenarios", Industrial Management \& Data Systems, Vol. 107 Iss: 6, pp.749 - 761.

[11] Bhushan Kapoor, Joseph Sherif, (2012) "Human resources in an enriched environment of business intelligence", Kybernetes, Vol. 41 Iss: 10, pp.1625 - 1637.

[12] Ask, (2010), MS Visio Definition [online] Avialable at http://www.ask.com/question/what-is-visioused-for [Accessed 26-Jan-2014].

[13] Martin, J; (1987), “The best practice of business. London”. John Martin Publishing.

[14] Hadfield, W. (2007), "BP to save 600 million pounds in global IT process standardisation", Computer Weekly, May, p. 5. 